\title{
Incidences of Deep Vein Thrombosis and Pulmonary Embolism after Total Knee Arthroplasty Using a Mechanical Compression Device with and without Low-Molecular-Weight Heparin
}

Sin Hyung Park, MD, Joong Hyeon Ahn, MD, Yong Bok Park, MD, Sun Geun Lee, MD, and Soo Jae Yim, MD Department of Orthopedic Surgery, Soonchunhyang University Bucheon Hospital, Bucheon, Korea

Purpose: To investigate the incidence of thromboembolic events and complications related to bleeding after total knee arthroplasty (TKA) with a mechanical compression device alone or in combination with low-molecular-weight heparin (LMWH).

Materials and Methods: A total of 489 TKA patients (776 knees) were retrospectively reviewed for the incidence of thromboembolic events and complications related to bleeding. While 233 patients ( 354 knees) were treated with a mechanical compressive device without LMWH, 256 patients ( 422 knees) were treated with the mechanical compressive device along with LMWH.

Results: The incidences of deep vein thrombosis (DVT) and pulmonary embolism (PE) were 15 of 375 knees (4.0\%) and 5 of $375 \mathrm{knees}(1.3 \%)$, respectively, in the group that used only a mechanical compressive device, and 14 of 401 knees (3.4\%) and 5 of 401 knees (1.2\%), respectively, in the group that used the mechanical compressive device with $\mathrm{LMWH}$. There was no significant difference between the two groups ( $\mathrm{p}=0.125$ and $\mathrm{p}=0.146$, respectively). The postoperative hemovac drainage amount was $635 \pm 57 \mathrm{~mL}$ in the group with a mechanical compressive device only and $813 \pm 84 \mathrm{~mL}$ in the group with the device and LMWH; therefore, the amount of drainage was significantly greater in the latter group ( $\mathrm{p}=0.013$ ).

Conclusions: Mechanical compression alone for prophylaxis against DVT and PE after TKA can be an attractive option in Korean patients.

Keywords: Knee, Arthroplasty, Thromboembolism, Prophylaxis, Mechanical, Intermittent pneumatic compression device

\section{Introduction}

Postoperative venous thromboembolism (VTE), including deep vein thrombosis (DVT) and pulmonary embolism (PE), is a lifethreatening complication in patients undergoing surgery. Total knee arthroplasty (TKA) has a high risk of DVT and $\mathrm{PE}^{1-3)}$, and because a thromboembolic event is fatal, routine thrombopro-

Received January 26, 2016; Revised (1st) March 28, 2016; (2nd) May 27, 2016; (3rd) June 30, 2016; Accepted July 16, 2016

Correspondence to: Soo Jae Yim, MD

Department of Orthopaedic Surgery, Soonchunhyang University Bucheon Hospital, 170 Jomaru-ro, Wonmi-gu, Bucheon 14584, Korea Tel: +82-32-621-5260, Fax: +82-32-621-5018

E-mail: yims@chol.com

This is an Open Access article distributed under the terms of the Creative Commons Attribution Non-Commercial License (http://creativecommons.org/licenses/by-nc/4.0/) which permits unrestricted non-commercial use, distribution, and reproduction in any medium, provided the original work is properly cited. phylaxis is recommended after $\mathrm{TKA}^{4-6)}$. Different modalities including the use of pharmacological agents and mechanical compression devices have been introduced with the aim of decreasing the prevalence of DVT and PE after TKA. Among those, pharmacological agents are usually used to prevent DVT, but they can increase the risk of bleeding complications ${ }^{7,8)}$. Mechanical compression devices have no risk of bleeding complications, but they are relatively less effective ${ }^{9-11)}$.

Some reports have described a low prevalence of DVT and PE after TKA in Asian patients compared with western populations ${ }^{12-14)}$. It is generally believed that the rate of VTE is low in Asia, so prophylaxis is seldom used ${ }^{15)}$. The differences in VTE prevalence have been attributed to differences in genetic background and dietary habits. In contrast, some reports have shown that dietary habits of Asians have changed similar to those of westerners and that the incidence of thromboembolic events has increased $^{16,17)}$.

If the occurrence of a thromboembolic event is low, prophy- 
laxis will result in unnecessary costs and complications related to bleeding. Therefore, whether pharmacological thromboprophylaxis is necessary to decrease the prevalence of DVT and PE after TKA in Asian patients is a legitimate question to raise. Mechanical compression thromboprophylaxis method may be sufficient for Asian patients.

The purpose of this study was to investigate the incidence of thromboembolic events (specifically, DVT and PE) and complications related to bleeding after TKA in patients treated with a mechanical compression device only and those treated with the same device in combination with a pharmacological agent.

We hypothesized that 1) there would be no statistically significant difference in the incidence of thromboembolic events including of DVT and PE between patients treated after TKA with mechanical compression only and those treated with compression and low-molecular-weight heparin (LMWH) and 2) bleeding-related complications would be less prevalent in those treated using mechanical compression only.

\section{Materials and Methods}

\section{Patients}

A total of 1,156 primary cemented TKAs were done in 745 patients from March 2009 to March 2015. Inclusion criteria were primary TKA using a mechanical compressive device with or without pharmacologic agents for prevention of DVT and PE. Exclusion criteria were the use of warfarin or aspirin before surgery for the treatment of other vascular, cardiac, or cerebral diseases and patients with any history of previous PE or DVT. We excluded 256 patients (380 knees) because they used warfarin or aspirin preoperatively and also for up to 48 hours following TKA for the aforementioned reasons. The remaining 489 patients (776 knees) were reviewed retrospectively. Of these, 233 patients (354 knees) used only a mechanical compression device without LMWH and 256 patients (422 knees) used the same mechanical compressive device along with LMWH. Of the 489 patients, 287 patients (574 knees) underwent bilateral TKA with a one-week interval between procedures and 202 patients underwent unilateral TKA. The 489 patients comprised 86 males and 403 females, with a mean age of $68.6 \pm 7.6$ years (range, 52 to 81 years) and mean body mass index (BMI) of $27.4 \mathrm{~kg} / \mathrm{m}^{2}$ (range, 23.0 to 30.5 $\mathrm{kg} / \mathrm{m}^{2}$ ). The average follow-up was 3.6 years (range, 2 to 5 years). All patients had osteoarthritis of the knee. The demographic data including age, sex, and BMI were compared between two groups. Histories related to thrombotic risk factors including smoking habits, congestive heart failure, and cancer were assessed and recorded. Preoperative coagulation assays (platelet count, prothrombin time, partial thromboplastin time, fibrinogen), full blood count, blood typing, and serum chemical profiling were performed for all patients.

\section{Surgical Procedure}

Surgeries were all performed by the same surgeon (SJY) using a medial parapatellar approach. The press-fit condylar TKA (Johnson \& Johnson, Raynham, MA, USA) or Scorpio NRG (Stryker Inc., Mahwah, NJ, USA) was used with tourniquet inflation to $350 \mathrm{mmHg}$. After a skin incision, a capsular incision was performed to expose the medial and lateral condyles, with subsequent tibial plateau and medial soft tissue release. A measured resection technique was used for the proximal tibial and distal femoral bone cuts. The tibial cut was made perpendicular to the mechanical axis using an extra-medullary alignment guide, with the posterior tibial slope set at $0^{\circ}$. After thoroughly removing posterior femoral osteophytes, a trial implant was inserted to assess the joint space, varus/valgus stability, patellar tracking, and condylar lift-off. Cement was used for tibial and femoral fixation. Patellar resurfacing was not performed in all knees. Tibial osteophyte removal and chondral shaving were performed. If there was synovial thickening observed in a given patient, a synovectomy was performed as well.

Straight leg raising exercises were initiated from the day of surgery. The drain was removed from the second postoperative day and continuous passive motion was started. From the first postoperative week, weight bearing ambulation was allowed.

\section{Thromboembolic Event Prophylaxis}

The DVT-3000 impulse system (DS Maref, Gunpo, Korea) was used on both legs postoperatively for mechanical compression prophylaxis of all patients. Mechanical compression was started on the day of the operation and continued for 10 to 14 days after the operation until the patients were discharged home. In the bilateral TKA patients, the mechanical compressive device was applied from the first operation day to one day before the second operation. We used sleeves with three circumferential air chambers running the distance from the ankle to the knee. Pharmacological thromboprophylaxis involved LMWH in all patients. These patients received a subcutaneous injection of LMWH in the form of enoxaparin 4000 IU (Sanofi-Aventis, Gentilly, France) beginning 24 hours after surgery ${ }^{18)}$.

\section{Assessment of Thromboembolic Events}

Color Doppler ultrasonography was performed for bilateral 
common femoral veins, superficial veins, popliteal veins, and calf veins by skilled physicians seven days after surgery in all patients.

$\mathrm{D}$-dimer levels were measured and PE and low extremity venography dynamic computed tomography were performed for patients suspected of DVT or PE. Symptoms of DVT and PE including chest pain, dyspnea, and painful swelling on lower extremities and positive findings on color Doppler ultrasonography were considered grounds for exclusion. For evaluation of bleeding tendency, the postoperative hemovac drainage amount and bleeding-related complications were checked and compared between the two groups. If hemoglobin was lower than $8 \mathrm{~g} / \mathrm{dL}$ or the patient showed hemodynamic instability, transfusion was performed.

\section{Statistical Analysis}

Differences of parameters including postoperative hemovac drainage, age, BMI, preoperative coagulation assay, full blood count, and serum chemical profile tests between both groups were assessed using Student $t$-test or Wilcoxon nonparametric test. The incidence of thromboembolic events and bleedingrelated complications between patients with and without PE were compared using chi-square and Fisher exact tests. Significance was set at $\mathrm{p}<0.05$. The statistical analyses were performed using SPSS ver. 12.0 (SPSS Inc., Chicago, IL, USA).

\section{Results}

There were no significant differences between the two groups in demographic characteristics and preoperative coagulation assay results (Table 1). The incidence of symptomatic DVT was 15 of 375 knees $(4.0 \%)$ in patients treated only using mechanical compression and 14 of 401 knees (3.4\%) in patients treated with the combination of mechanical compression and LMWH. The prevalence of symptomatic PE was 5 of 375 knees (1.3\%) in patients treated with mechanical compression alone and 5 of 401 knees $(1.2 \%)$ in those treated with compression and LMWH.

Table 1. Demographics and Preoperative Coagulation Assay of Each Group

\begin{tabular}{|c|c|c|c|}
\hline Parameter & $\begin{array}{l}\text { Mechanical compression } \\
\text { only group }(n=233)\end{array}$ & $\begin{array}{c}\text { Mechanical compression and } \\
\text { pharmacological agent group }(n=256)\end{array}$ & p-value \\
\hline Age (yr) & $64.3 \pm 10.7$ & $68.4 \pm 11.4$ & $0.174^{\mathrm{a})}$ \\
\hline Sex $(M: F)$ & $35: 49$ & $50: 74$ & $0.164^{\mathrm{b})}$ \\
\hline Body mass index $\left(\mathrm{kg} / \mathrm{m}^{2}\right)$ & $26.2 \pm 3.0$ & $27.9 \pm 2.7$ & $0.153^{\mathrm{a})}$ \\
\hline \multicolumn{4}{|l|}{ Preoperative coagulation assay } \\
\hline Prothrombin time international normalized ratio & $1.14 \pm 0.4$ & $1.10 \pm 0.3$ & $0.156^{\mathrm{a})}$ \\
\hline Activated partial prothrombin time (sec) & $24.4 \pm 7.3$ & $27.0 \pm 7.1$ & $0.135^{\mathrm{a})}$ \\
\hline Platelet count $\left(10^{3}\right)$ & $215 \pm 87$ & $232 \pm 79$ & $0.154^{\mathrm{a})}$ \\
\hline
\end{tabular}

Values are presented as mean \pm standard deviation.

${ }^{\text {a) }}$ Student $t$-test. ${ }^{\text {b) }}$ Chi-squre test.

Table 2. Incidence of Thromboembolic Events in Each Group

\begin{tabular}{cccc}
\hline Complication & $\begin{array}{c}\text { Mechanical compression } \\
\text { only group }(\mathrm{n}=233)\end{array}$ & $\begin{array}{c}\text { Mechanical compression and } \\
\text { pharmacological agent group }(\mathrm{n}=256)\end{array}$ & $\begin{array}{c}\mathrm{p} \text {-value } \\
\text { Deep vein thrombosis (\%) }\end{array}$ \\
Pulmonary embolism (\%) & $5(4)$ & $5(1.2)$ & 0.125 \\
\hline
\end{tabular}

Table 3. Complications Related to Bleeding in Each Group

\begin{tabular}{|c|c|c|c|}
\hline Complication & $\begin{array}{l}\text { Mechanical compression } \\
\text { only group }(\mathrm{n}=233)\end{array}$ & $\begin{array}{l}\text { Mechanical compression and } \\
\text { pharmacological agent group }(n=256)\end{array}$ & p-value \\
\hline Hematoma (\%) & $0(0)$ & $4(2)$ & $0.024^{\mathrm{a})}$ \\
\hline Postoperative hemovac drainage $(\mathrm{mL})$, mean \pm SD & $635 \pm 57$ & $813 \pm 84$ & $0.013^{\mathrm{b})}$ \\
\hline
\end{tabular}

SD: standard deviation.

${ }^{\text {a) }}$ Chi-squre test. ${ }^{\text {b) }}$ Student $t$-test. 
The incidences of DVT and PE were not significantly different between the groups (Table 2). Postoperative hemovac drainage amount was $635 \pm 57 \mathrm{~mL}$ and $813 \pm 84 \mathrm{~mL}$ in the respective group, indicating a significant difference (Table 3). Thrombi that developed in 39 knees resolved completely regardless of their site or size without pharmacological treatment as assessed by sonogram and venogram performed 6 months postoperatively. No patient had symptomatic PE as indicated by the lack of abnormal findings on perfusion lung scan and the absence of symptoms.

\section{Discussion}

One of the principle findings of this study is the lack of statistical differences in the incidence of thromboembolic events including DVT and PE between treatment with a mechanical compressive device alone and compression delivered along with LMWH after TKA. Another principle finding is the greater bleeding tendency in terms of postoperative hemovac drainage in patients treated with the combination approach (i.e., greater bleeding after the use of LMWH).

There are LMWH, rivaroxaban, fondaparinux, warfarin, and aspirin in pharmacological methods for prophylaxis of DVT and PE. LMWH inhibits factor Xa. The major advantage of LMWH is that no monitoring is required, but it needs to be administered via a subcutaneous injection. Rivaroxaban is a direct Xa inhibitor that is taken orally and requires no monitoring. But rivaroxaban was associated with a higher rate of reoperation than was LMWH after $\mathrm{TKA}^{19)}$. Fondaparinux is a synthetic pentasaccharide and an indirect factor Xa inhibitor. The drug has had limited use in North America because of concerns related to bleeding ${ }^{18)}$.

Nagase et al. ${ }^{20)}$ reported that the prevalence of postoperative PE after the use of mechanical prophylaxis alone was $0.66 \%$, which was similar to $0.40 \%$ after the combined use of fondaparinux and mechanical prophylaxis in patients with total hip arthroplasty or TKA. Kim et al. ${ }^{21)}$ reported that the prevalence of DVT was 94 of 1434 knees $(6.6 \%)$ in patients with a mechanical compression device after TKA. There was a low prevalence of DVT and PE in Asian patients, and all thrombi resolved completely and spontaneously without treatment using anticoagulants in a study by Kim et al. ${ }^{21)}$. Kanchanabat et al. ${ }^{22)}$ performed a systematic review and meta-analysis that addressed the rate of VTE after orthopedic surgery in Asian patients without thromboprophylaxis. The pooled rates of proximal and symptomatic DVT were lower in Asians than those in western reports and none of these Asian patients with orthopedic surgery died from VTE. Especially, the prevalence of DVT in Koreans was lower than that in other
Asians (Korean, 7.5\%; South-East Asian, 11.8\%; and Japanese, $11.0 \%)^{22)}$.

The present study compared the incidence of DVT and PE between patients treated using only a mechanical compressive device and those treated using the device along with $\mathrm{LMWH}$ after TKA. This comparison has not been done before, except in a systematic review ${ }^{22)}$. In our study, the incidences of symptomatic DVT and PE were 15 of 375 knees (4.0\%) and 5 of 375 knees $(1.3 \%)$ in patients treated with mechanical compression alone. There was no significant difference compared to the group with the combined use of a mechanical compressive device and LMVH where the incidences were 14 of 401 knees (3.4\%) and 5 of 401 knees (1.2\%). These incidences are similar to those described in the previous systematic review study $(4.5 \% \text { and } 0.6 \%)^{22}$. There were some limitations in our study. It was a retrospective study, not a prospective randomized study, subject to selection bias. However, to the best of our knowledge, there has been no randomized controlled trial conducted on this subject; therefore, a randomized prospective study needs to be performed. Second, the number of patients was relatively small considering the low incidences of DVT and PE. Because the incidences of DVT and PE were low, more patients will be needed to achieve statistical significance. Third, the study involved only Korean patients. Therefore, the results may not be applicable to other ethnic groups. Fourth, we used only LMWH as the pharmacological agent. Therefore, the group treated with a mechanical compressive device and LMWH in our study does not represent groups that use other pharmacological agents. Fifth, we did not include other groups for comparison such as a group that used only pharmacological agent or a group that used neither a pharmacological agent nor a mechanical compressive device. However, we aimed to investigate if the use of a mechanical compression device alone would be sufficient to prevent thromboembolic events after TKA in Asians. In our patients, the additional use of a pharmacological agent did not make a difference in prevention of thromboembolic events, and rather the bleeding-related complication rate was lower in the group with compression treatment only. Therefore, we think the comparison was appropriate for the study purpose.

\section{Conclusions}

Mechanical compression after TKA resulted in a low prevalence of DVT and PE, similar to the combined use of mechanical compression and LMWH as a pharmacological agent. Complications related to bleeding occurred less often in patients treated only using mechanical compression. Therefore, the use of mechanical 
compression only for prophylaxis against DVT and PE can be an attractive option for Korean patients.

\section{Conflict of Interest}

No potential conflict of interest relevant to this article was reported.

\section{References}

1. Parvizi J, Sullivan TA, Trousdale RT, Lewallen DG. Thirtyday mortality after total knee arthroplasty. J Bone Joint Surg Am. 2001;83:1157-61.

2. Howie C, Hughes H, Watts AC. Venous thromboembolism associated with hip and knee replacement over a tenyear period: a population-based study. J Bone Joint Surg Br. 2005;87:1675-80.

3. Cohen AT. Long-term benefits of preventing venous thromboembolic events. Curr Med Res Opin. 2012;28:877-89.

4. McRae SJ, Ginsberg JS. Initial treatment of venous thromboembolism. Circulation. 2004;110(9 Suppl 1):I3-9.

5. Eikelboom JW, Karthikeyan G, Fagel N, Hirsh J. American Association of Orthopedic Surgeons and American College of Chest Physicians guidelines for venous thromboembolism prevention in hip and knee arthroplasty differ: what are the implications for clinicians and patients? Chest. 2009;135:513-20.

6. Weinmann EE, Salzman EW. Deep-vein thrombosis. N Engl J Med. 1994;331:1630-41.

7. Colwell CW Jr, Froimson MI, Anseth SD, Giori NJ, Hamilton WG, Barrack RL, Buehler KC, Mont MA, Padgett DE, Pulido PA, Barnes CL. A mobile compression device for thrombosis prevention in hip and knee arthroplasty. J Bone Joint Surg Am. 2014;96:177-83.

8. Hull RD, Yusen RD, Bergqvist D. State-of-the-art review: assessing the safety profiles of new anticoagulants for major orthopedic surgery thromboprophylaxis. Clin Appl Thromb Hemost. 2009;15:377-88.

9. Pidala MJ, Donovan DL, Kepley RF. A prospective study on intermittent pneumatic compression in the prevention of deep vein thrombosis in patients undergoing total hip or total knee replacement. Surg Gynecol Obstet. 1992;175:47-51.

10. Woolson ST, Watt JM. Intermittent pneumatic compression to prevent proximal deep venous thrombosis during and after total hip replacement: a prospective, randomized study of compression alone, compression and aspirin, and compression and low-dose warfarin. J Bone Joint Surg Am. 1991;73:507-12.

11. Kaempffe FA, Lifeso RM, Meinking C. Intermittent pneumatic compression versus coumadin: prevention of deep vein thrombosis in lower-extremity total joint arthroplasty. Clin Orthop Relat Res. 1991;(269):89-97.

12. Fuji T, Ochi T, Niwa S, Fujita S. Prevention of postoperative venous thromboembolism in Japanese patients undergoing total hip or knee arthroplasty: two randomized, doubleblind, placebo-controlled studies with three dosage regimens of enoxaparin. J Orthop Sci. 2008;13:442-51.

13. Kim KI, Cho KY, Jin W, Khurana SS, Bae DK. Recent Korean perspective of deep vein thrombosis after total knee arthroplasty. J Arthroplasty. 2011;26:1112-6.

14. Leizorovicz A, Turpie AG, Cohen AT, Wong L, Yoo MC, Dans A; SMART Study Group. Epidemiology of venous thromboembolism in Asian patients undergoing major orthopedic surgery without thromboprophylaxis: the SMART study. J Thromb Haemost. 2005;3:28-34.

15. Cohen AT, Tapson VF, Bergmann JF, Goldhaber SZ, Kakkar AK, Deslandes B, Huang W, Zayaruzny M, Emery L, Anderson FA Jr; ENDORSE Investigators. Venous thromboembolism risk and prophylaxis in the acute hospital care setting (ENDORSE study): a multinational cross-sectional study. Lancet. 2008;371:387-94.

16. Fujita S, Hirota S, Oda T, Kato Y, Tsukamoto Y, Fuji T. Deep venous thrombosis after total hip or total knee arthroplasty in patients in Japan. Clin Orthop Relat Res. 2000;(375):16874.

17. Sudo A, Sano T, Horikawa K, Yamakawa T, Shi D, Uchida A. The incidence of deep vein thrombosis after hip and knee arthroplasties in Japanese patients: a prospective study. J Orthop Surg (Hong Kong). 2003;11:174-7.

18. Lieberman JR, Pensak MJ. Prevention of venous thromboembolic disease after total hip and knee arthroplasty. J Bone Joint Surg Am. 2013;95:1801-11.

19. Jensen CD, Steval A, Partington PF, Reed MR, Muller SD. Return to theatre following total hip and knee replacement, before and after the introduction of rivaroxaban: a retrospective cohort study. J Bone Joint Surg Br. 2011;93:91-5.

20. Nagase Y, Yasunaga H, Horiguchi H, Hashimoto H, Shoda N, Kadono Y, Matsuda S, Nakamura K, Tanaka S. Risk factors for pulmonary embolism and the effects of fondaparinux after total hip and knee arthroplasty: a retrospective observational study with use of a national database in Japan. J Bone Joint Surg Am. 2011;93:e146. 
21. Kim YH, Kulkarni SS, Park JW, Kim JS. Prevalence of deep vein thrombosis and pulmonary embolism treated with mechanical compression device after total knee arthroplasty in Asian patients. J Arthroplasty. 2015;30:1633-7.

22. Kanchanabat B, Stapanavatr W, Meknavin S, Soorapanth
C, Sumanasrethakul C, Kanchanasuttirak P. Systematic review and meta-analysis on the rate of postoperative venous thromboembolism in orthopaedic surgery in Asian patients without thromboprophylaxis. Br J Surg. 2011;98:1356-64. 